\title{
Optimal Rendezvous Sequence for LEO Debris Capture
}

\author{
Ciro Borriello ${ }^{1}$ and Lorenzo Casalino ${ }^{2}$ \\ 1. Aviospace (Airbus Defence \& Space), Via Botero 18, Torino 10122, Italy \\ 2. Department of Mechanical \& Aerospace Engineering, Politecnico di Torino, Torino 10129, Italy
}

Received: January 24, 2014 / Accepted: January 07, 2015 / Published: January 30, 2015.

\begin{abstract}
The primary purpose of this study is to exploit the effect of Earth's non-sphericity perturbation, particularly due to the $J_{2}$ term, in order to optimize the capture sequence of potential orbital debris, that is the cumulative $\Delta V$ associated to the transfers between one object and the others. As results of several researches and model predictions, many international agencies agree that the growing population of objects and debris in LEO (low earth orbits), will follow a diverging trend in the future. This, in turn, would constitute a serious threat to circum-terrestrial space safety and sustainability. In LEO, the $J_{2}$ disturbance is prevailing over the others, and it acts by affecting the longitude of the ascending node $(\Omega)$, the argument of perigee $(\omega)$ and, accordingly, the true anomaly (v). Therefore, the goal of optimizing the $\Delta V$ is achieved by taking advantage of the rate of variation of $\Omega$ and $\omega$, thereby compensating for the $\Delta \Omega$ and $\Delta \omega$, present between the orbital transfer vehicle (chaser) and the debris to be captured (target). Obviously, the perturbation will lead to favourable variations of the orbital parameters only for some combinations of $\Omega$ and $\omega$. Yet the presence of a debris population with random distribution of $\Omega$ and $\omega$, makes this application particularly suited to the problem. The single maneuver has been modelled with a 4-impulse time fixed rendezvous and the optimization problem has been addressed by implementing a hybrid evolutionary algorithm, which adopts, in parallel, three different strategies, namely, genetic algorithm, differential evolution and particle swarm optimization.
\end{abstract}

Key words: Trajectory optimization, hybrid evolutionary algorithms, debris removal, multi-target rendezvous, $J_{2}$ perturbation assist.

\section{Introduction}

The steady increase in the number of debris in LEO (low earth orbit) is a matter of great interest and concern in the scientific community [1], as the problem regards the future safety and stability of the exploitation of circum-terrestrial space. Nowadays, launching new artificial satellites and spacecrafts without worrying about their future disposal and de-orbiting, as well as without planning the end of their operational life cycle, is no longer a viable approach. On the other hand, intervening only on "end of life" strategies may not be enough. The number of objects in orbit rapidly increased over the last decades (Fig. 1) and the number of collisions and the resulting trail of debris that these events produce are likely to trigger a chain process, whose effects are difficult to

\footnotetext{
Corresponding author: Ciro Borriello, M. Sc., research filed: Aerospace Engineering. E-mail: ciro.borriello@aviospace.com.
}

predict. Internal research of top aerospace agencies (e.g., NASA (National Aeronautics and Space Administration), ESA (European Space Agency), JAXA (Japan Aerospace Exploration Agency)) [2] already addressed the design and analysis of ADR (active debris removal) missions, needed to face the orbital debris threat. Top-level timeline includes the following cyclic operations: target debris rendezvous; close approach operations; non-cooperative de-tumbling; docking; de-orbiting; new target debris selection and rendezvous.

Each phase of the mission needs to be successful, and research needs to move important steps and evolutions towards different subject areas. In particular, it is essential to maximize the number of targets that a certain orbital transfer vehicle (chaser) is able to capture and de-orbit, to specify algorithms for orbital transfer calculation, and to optimize the debris sequence efficiency. Moreover, 
during the target approach phase, 3D vision algorithms shall allow an accurate $3 \mathrm{D}$ reconstruction of target inertial properties and dynamics, in order to execute a safe capture. Finally, non-cooperative docking will require a completely autonomous and reliable relative navigation system to be developed.

The present study arises from these needs and focuses on the rendezvous sequence of non-cooperative objects. The orbital slot of the greatest interest is the LEO environment, containing debris of larger dimensions, such as upper stages and inactive satellites, with masses exceeding one ton. Since the problem is most critical in the LEO slot, an assessment of the perturbations in this area was first conducted.

The $J_{2}$ perturbation due to Earth's non-sphericity is predominant, thus it is possible to exploit its effects in order to optimize the energy required for transfers between one target and the other. Such disturbances affect only the longitude of ascending node $(\Omega)$, the argument of perigee $(\omega)$ and the true anomaly $(v)$. With the availability of a wide target population, the rendezvous sequence will be chosen so that the rate of variation of orbital parameters affected by the $J_{2}$ effect tends to align the chaser and target. Hence, it is possible to save the $\Delta V$ needed to compensate for $\Delta \Omega$ and $\Delta \omega$ between target and chaser.

For the calculation of $\Delta V$ associated with each sequence step, a 4-impulse time fixed rendezvous maneuver has been modelled, and the optimization parameters have been evaluated by means of a hybrid evolutionary algorithm. The results obtained have been compared with those produced by the same algorithm, nullifying the contribution of the $J_{2}$ disturbance, and highlighting the gain obtained in terms of $\Delta V$.

Hand in hand with the active debris removal missions, the study of optimal rendezvous strategies with multiple non-cooperative objects can be very useful for other purposes such as servicing and refuelling inactive satellites willing to prolong their operational life cycle.

\section{Orbital Debris Hazard and Mitigation Strategies}

The space activity, since its inception, has always given a relatively low value to disposal of satellites and orbiting objects, once the operational life cycle reaches the end. Only in recent decades, necessity led to draw up precise guidelines with the aim of limiting the number of debris in Earth orbits (from LEO to GEO (geosynchronous equatorial orbit)). Yet the risk inherent in the presence of an increasing number of objects in orbit tends to worsen as emerging nations acquire the technology needed to exploit the circum-terrestrial space. Currently, the overall number of objects in LEO with characteristic dimensions above $10 \mathrm{~cm}$ is more than 10,000 .

Approximately one-sixth of the causes of fragmentation of objects orbiting the Earth are still unknown. However, most of them can be identified among: deliberate actions; propulsive causes; batteries issues; collisions; aerodynamic drag.

Debris fragmentation by collision is nowadays still restrained, though over the next century it is expected to be the leading and no longer controlled cause of generation of debris in LEO. Ref. [3] show that even with no future launches of objects in orbit, collisions between those already present would lead to a continually growing number of debris.

The uncontrolled growth of debris in LEO may be limited only by reducing the number of collisions. To avoid a catastrophic collision chain there are only two solutions: de-orbiting and disposal on alternative orbits.

Over the last decade, the international community agreed to adopt rules specifically aimed at defining a PMD (post mission disposal) strategy. These guidelines recommend that any payload or upper stage was removed from its operational orbit within 25 years after the end of his life cycle. Yet considering the current orbital environment, even if all new satellites followed the PMD guidelines completely (100\%), this would not reduce the risk of a collision cascade. Hence, 


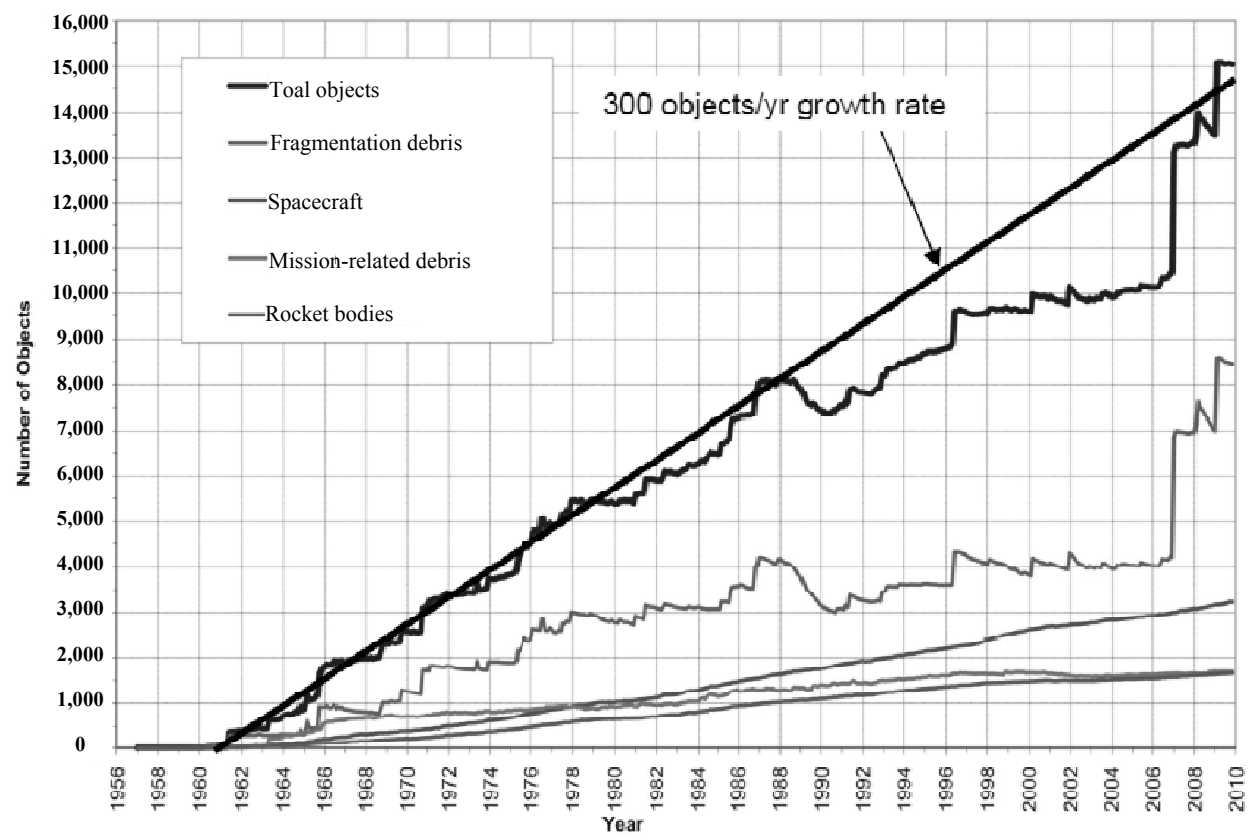

Fig. 1 LEO and GEO orbital debris population, classified on the basis of debris source [2].

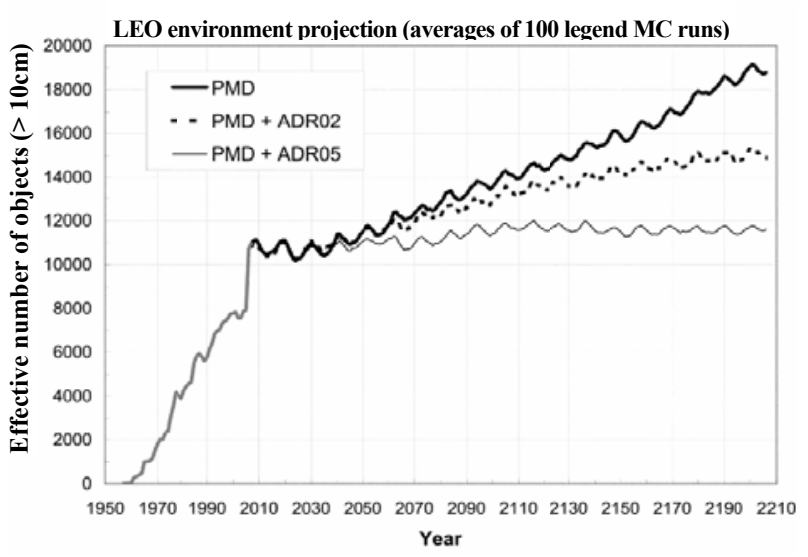

Fig. 2 LEO environment NASA projections and growth trends, according to legend model. Three cases are represented, with different combinations of PMD and ADR.

the adoption of a plan of ADR is strongly suggested [4]. Fig. 2 shows the trend forecasts calculated with the LEGEND model, developed by NASA [5]:

The following three case studies have been taken into consideration:

(1) PMD at $90 \%$;

(2) PMD at $90 \%+$ two removed objects per year;

(3) PMD at $90 \%+$ five removed objects per year.

Of course, if it is impossible to reach $90 \%$ compliance with PMD, it would be required a higher rate of ADR in order to obtain a stable environment. Finally, by equipping future payload devices with ACA (active collision avoidance) systems, it is possible to act in synergy with the PMD and ADR and achieve the common goal of making the orbital environment less risky for future missions.

\section{Single Target Rendezvous Optimization}

The ultimate goal is to minimize the $\Delta V$ associated with a sequence of rendezvous maneuvers in LEO, not necessarily circular and/or coplanar, and sometimes very close to each other. Each single rendezvous has been modelled as a 4-impules time fixed maneuver, as shown in Fig. 3:

The entire maneuver can be split into three arcs and four impulses, defined by the variation of velocity vector and the angle of shot. $t_{0}$ is the maneuver start time, whereas $t_{3}$ is the instant at which rendezvous occurs. The difference $T_{\mathrm{fi}}=t_{3}-t_{0}$ is the maneuver time of flight, defined as input to the optimization algorithm. $R_{i}$ and $R_{f}$ are the position vectors of the chaser and target on their orbits, respectively. Thus at the end of the maneuver, the chaser will transfer from $R_{i}$ to $R_{f}$. The optimization program (Fortran-based 


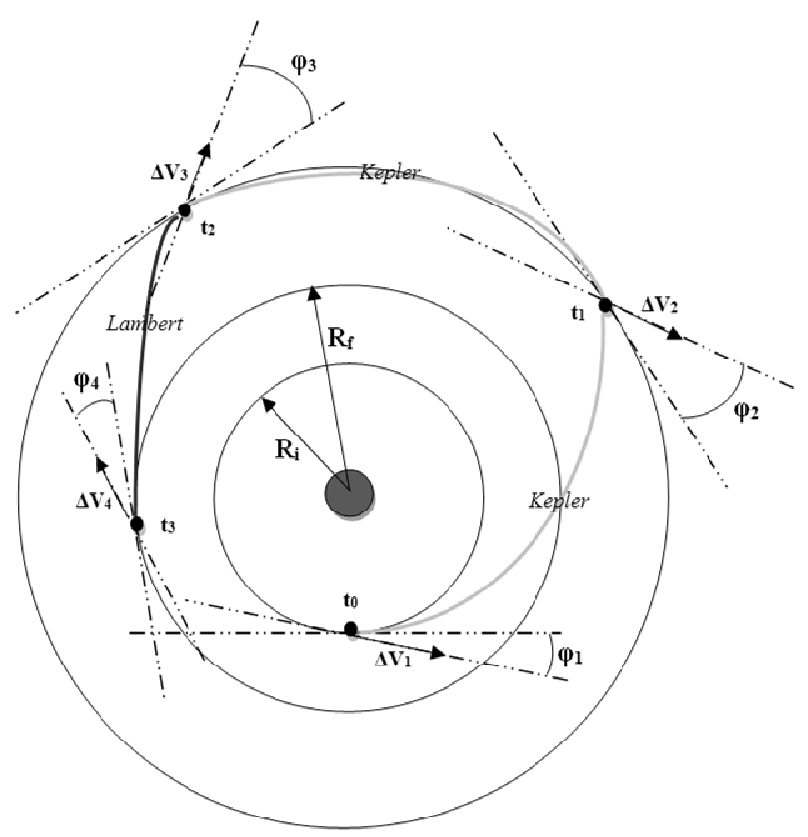

Fig. 3 4-impulses time-fixed rendezvous maneuver scheme, the whole transfer is split in three arcs.

language) used to find the optimal solution, which minimizes the $\Delta V$ associated with the rendezvous, is a HEA (hybrid evolutionary algorithm) based on the cooperation among three different strategies: GA (genetic algorithm), DE (differential evolution), PSO (particle swarm optimization). Recent studies [6,7] confirmed that, for rendezvous optimization problems, it may be convenient to adopt a hybridization strategy. In this case, balancing the strengths of the three EA (evolutionary algorithms) in parallel, considerable advantages can be obtained, in terms of convergence speed and optimization efficiency. In fact, in the hybrid procedure, any algorithm acts on a different population, and the best individual solutions are allowed to migrate from one population to another. The migration takes place only at predefined intervals within the iterative sequence. Algorithms are all initialized with random populations of solutions and then, following the evaluation of a specific fitness function, the best solutions are exchanged and crossed between the different selection strategies. In order to successfully implement the HEA strategy and optimize a single 4-impulse time fixed rendezvous, it is first necessary to define the useful components of the solution vector. These dimensionless components will uniquely characterize the maneuver. In the case considered, the solution vector will be defined by:

$$
\begin{gathered}
\text { POPULATION }= \\
{\left[t_{1}-t_{0}, t_{3}-t_{2}, \Delta V_{1}, \Delta V_{2}, \varphi_{1}, \varphi_{2}\right]}
\end{gathered}
$$

Furthermore, the following dimensionless input vector shall be provided for the chaser and the target:

$$
\mathbf{P O}=\left[a, e, \Omega+\omega, T_{i n}, i, \omega\right]
$$

The following flow-chart summarizes the steps undertaken by the optimization program, leading to the overall $\Delta V$ calculation.

The hybrid evolutionary algorithm assigns random values to the initial POPULATION array, thus $\Delta V_{1}, \varphi_{1}$ and $t_{1}-t_{0}$ are included in the first generation outputs. At this point, velocity components, immediately after the first burn, can be directly derived:

$$
\overrightarrow{V_{0}^{+}}=\overrightarrow{V_{0}}+\overrightarrow{\Delta V_{1}}
$$

Since $V_{0}^{-}$and $t_{1}-t_{0}$ are known, a dedicated subroutine is used to solve the Kepler problem on the first arc. Outputs are expressed in the form of the velocity vector $V_{1}^{-}$and the position $r_{1}$ at time $t_{1}$. From the first generation of POPULATION array, $\Delta V_{2}, \varphi_{2}$ and $t_{2}-t_{1}$ are known. Therefore, velocity vector after the second burn is given by:

$$
\overrightarrow{V_{1}^{+}}=\overrightarrow{V_{1}^{\longrightarrow}}+\overrightarrow{\Delta V_{2}}
$$

Likewise, another Kepler problem is solved, in order to compute the velocity vector $V_{2}^{-}$at the end of the second maneuver arc and at $r_{2}$ position. Since time of flight is known, it is now possible to derive the time associated with the last maneuver arc:

$$
\begin{aligned}
t_{3}-t_{2}= & T_{f i}-\left(t_{2}-t_{1}\right)-\left(t_{1}-t_{0}\right) \\
& =T_{f i}-t_{2}+t_{0}
\end{aligned}
$$

where, $t_{0}$ is the maneuver start time. Finally, since initial and final position on the third maneuver arc are known by means of $r_{2}$ and $r_{3}$, Lambert problem is solved, producing outputs of the velocity vectors $V_{2}^{+}$and $V_{3}^{-}$at orbital positions $r_{2}$ and $r_{3}$, respectively. It is now possible to compute the last two burns magnitudes: 


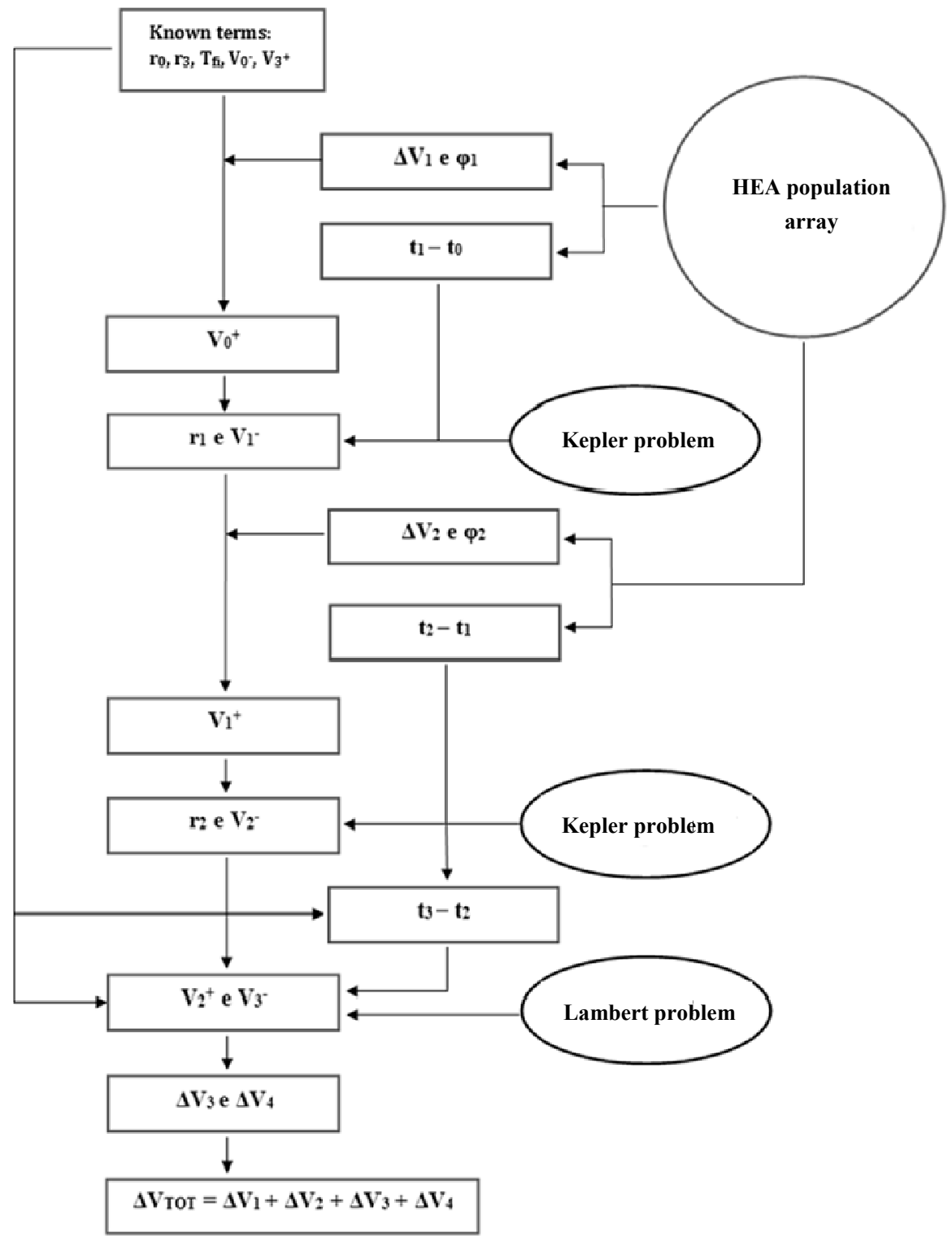

Fig. 4 HEA (hybrid evolutionary algorithm) optimization process flow chart.

$$
\begin{aligned}
& \Delta V_{3}=\left|V_{2}^{+}-V_{2}^{-}\right| \\
& \Delta V_{4}=\left|V_{3}^{+}-V_{3}^{-}\right|
\end{aligned}
$$

Global $\Delta V$ associated with the 4-impulses time fixed rendezvous maneuver is then obtained by summing up the single burns contributes:

$$
\Delta \boldsymbol{V}_{\text {тот }}=\Delta V_{1}+\Delta V_{2}+\Delta V_{3}+\Delta V_{4}
$$

The HEA optimizer fitness function is directly linked to the $\Delta V_{\text {Tот }}$ parameter. In fact, solutions with lower $\Delta V_{\text {TOT }}$ will be given a higher score and be encouraged in the iterative evolutionary process. HEA's driving parameters can be summarized in
Table 1:

$J_{2}$ perturbation effect has been introduced only on the first two arcs, managed by solving the Kepler problem, whereas the last arc duration may be variable, depending on the type of solver adopted. Best solutions have been selected between two different strategies adopted at this stage: single-revolution and multiple-revolution last arc, managed by solving the Lambert problem.

The objective is then to exploit the $J_{2}$ effect, by compensating for the $\Delta \Omega$ and $\Delta \omega$ between chaser and 
Table 1 HEA set-up parameters.

\begin{tabular}{|c|c|c|}
\hline Parameter & Description & HEA values \\
\hline NTOT & $\begin{array}{l}\text { Initialization } \\
\text { solutions }\end{array}$ & 490 \\
\hline TFI & Time of flight & $V($ variable $)$ \\
\hline $\mathrm{PO}$ & Input vectors & {$\left[a, e, \Omega+\omega, T_{i n}, i, \omega\right]$} \\
\hline Population & Solution vector & $\begin{array}{l}{\left[t_{1}-t_{0}, t_{3}\right.} \\
\left.-t_{2}, \Delta V_{1}, \Delta V_{2}, \varphi_{1}, \varphi_{2}\right]\end{array}$ \\
\hline NP & $\begin{array}{l}\text { POPULATION } \\
\text { dimension }\end{array}$ & 6 (1-target), 7 ( $N$-target) \\
\hline NG & $\begin{array}{l}\text { Number of } \\
\text { iterations }\end{array}$ & 200 \\
\hline PARMIN & $\begin{array}{l}\text { POPULATION } \\
\text { min values }\end{array}$ & {$\left[0,0,0,0,-3.5,-3.5,2^{*}\right]$} \\
\hline PARMAX & $\begin{array}{l}\text { POPULATION } \\
\text { max values }\end{array}$ & $\begin{array}{l}{[V, V, 0.3,0.3,3.5,3.5, N} \\
\left.+1^{*}\right]\end{array}$ \\
\hline NIGE & $\begin{array}{l}\text { GE initialization } \\
\text { solutions }\end{array}$ & $300(61 \%)$ \\
\hline NIDE & $\begin{array}{l}\text { DE initialization } \\
\text { solution }\end{array}$ & $160(33 \%)$ \\
\hline NIPSO & $\begin{array}{l}\text { PSO } \\
\text { initialization } \\
\text { solutions }\end{array}$ & $30(6 \%)$ \\
\hline
\end{tabular}

*In a 7D POPULATION vector, the 7th dimension refers to potential targets.

target. The effect is maximized by tuning the initial PO parameter. Let $\mathrm{PO}_{\mathrm{CHASER}_{0} 0}$ and $\mathrm{PO}_{\text {TARGET } 0}$ be the initial parameters for which, without perturbation effect, the optimization program produces an associated cost of $\Delta V_{\text {HOHMANN_o. When }} J_{2}$ perturbation is on, the cost increases to: $\Delta V_{\text {PERTURBED_0 }}>$ $\Delta V_{\text {HOHMANN_0. }}$.

In order to maximize the perturbation effect, it may be useful to track the rates of $\Delta \Omega$ and $\Delta \omega$ and therefore adjust the initial PO. Thence, by properly choosing initial PO and running a new simulation, it can be found that perturbation effects now acts aligning the final $\mathrm{PO}$, in order to have:

$\Delta V_{\text {PERTURBED_1 }} \approx \Delta V_{\text {HOHMANN_0 }}<\Delta V_{\text {HOHMANN_1 }}$

Where $\Delta V_{\text {HOHMANN_1 }}$ is the cost obtained with adjusted initial PO and $J_{2}$ perturbation off.

\section{Debris Capture Application}

The orbital parameters of potential debris can be mapped and graphed on the $\Omega-\omega$ plane, using the NORAD real-time database [8].

A random distribution of these parameters, affected by $J_{2}$, is favorable for the optimization problem. In fact, in a casual targets population, it results easier to find a proper combination of $\Omega$ and $\omega$, which maximizes the perturbation effect.

Next step is now to optimize $\Delta V$ associated with a single rendezvous maneuver between two objects in LEO, neither circular nor co-planar. Two sets of simulations have been carried on, both in the ideal and perturbed case. $\Delta V$ curves, function of time of flight, have been reported in the following graph:

Please note that $T_{f i}$ and $\Delta V$ are dimensionless parameters, with respect to:

$$
\sqrt{\frac{R_{T}^{3}}{\mu}} \text { for } T_{f i} \text { and } \sqrt{\frac{\mu}{R_{T}}} \text { for } \Delta V
$$

The oscillatory behavior of both curves is directly related to the formulation of the optimization parameters $\Delta V_{1}$ and $\Delta V_{2}$. In fact, these two burns are given in the chaser initial plane, whereas only the components $\Delta V_{3}$ and $\Delta V_{4}$ are those contributing to the change of plane and alignment with the target. Sometimes the position obtained after the first two impulses is relatively advantageous and leads to low cost options for the last two impulses. In other cases, it will be necessary to apply more expensive $\Delta V_{3}$ and $\Delta V_{4}$, which lead to local $\Delta V$ peaks associated with the overall maneuver.

For short times of flight the perturbation effect does not involve any advantage, since the absolute changes of $\Omega$ and $\omega$ are too small to be exploited in the optimization of $\Delta V$. However, for higher times of

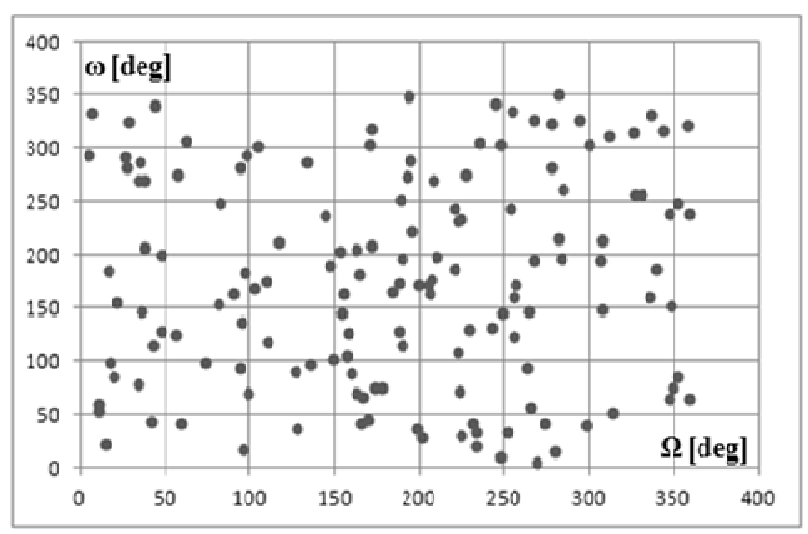

Fig. $4 \Omega$ and $\omega$ distribution for large debris (with high hazard associated to them) in LEO population. 


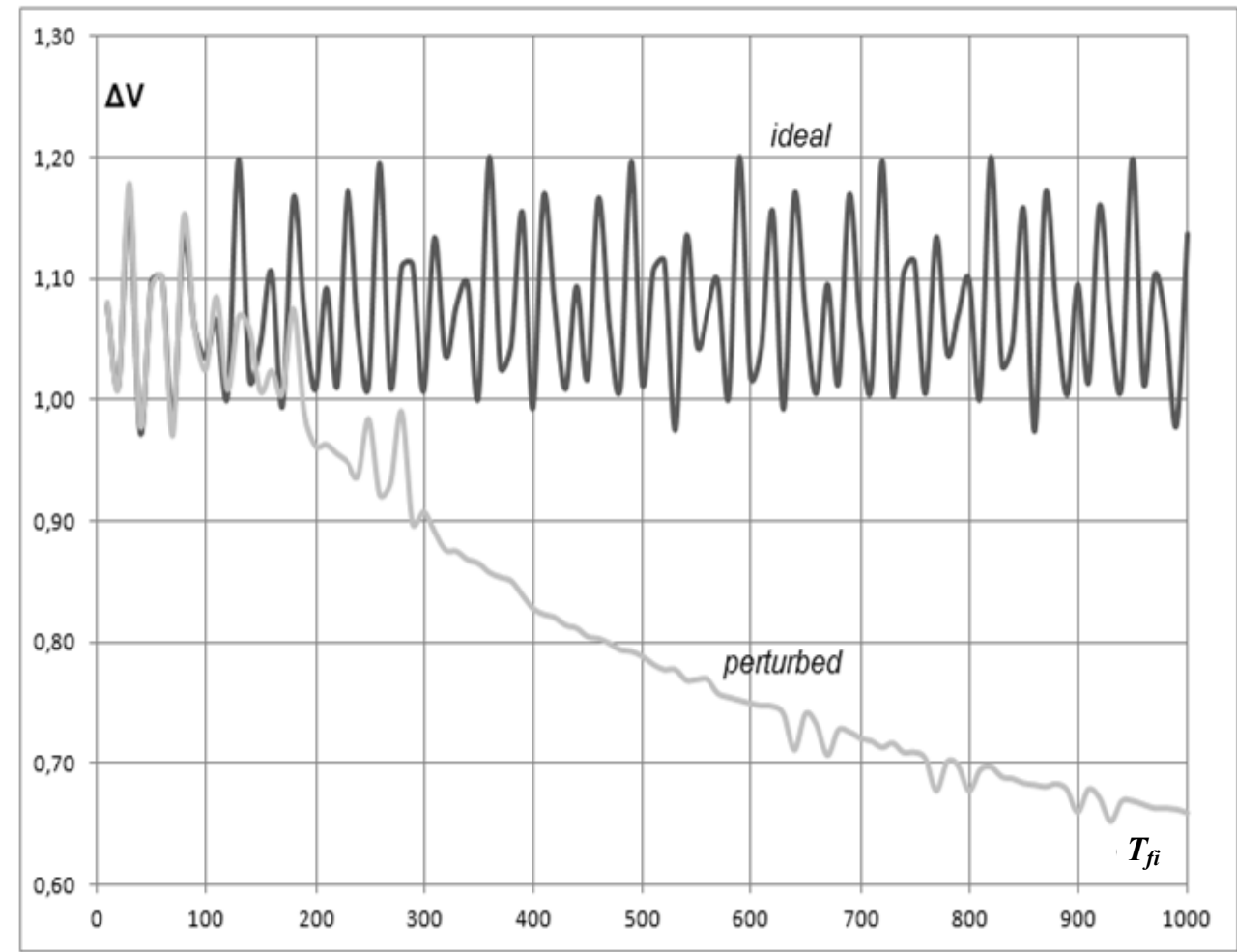

Fig. 5 Single target rendezvous energy (in terms of $\Delta V$ ) for the ideal and $J_{2}$ perturbed case.

flight, it is interesting to note that the $\Delta V$ associated with the transfer sensibly decreases. In this scenario, it is possible to accumulate quite consistent $\Delta \Omega$ and $\Delta \omega$ for both the chaser and the target. Consequently, the optimizer will tend to choose solutions in which the chaser moves on intermediate transfer orbits that lead to values of $\Delta \omega$ and $\Delta \Omega$ big enough to compensate for the gap with the target. By conducting further simulations, a negative slope of the curve in the perturbed case is obtained until it reaches an asymptotic value corresponding to the minimum $\Delta V$ required for the transfer between the two objects. In the sample case, this value is achieved with a time of flight greater than 8,000 . Values of this kind are however not applicable to debris capture missions, which aim at removing at least tens of objects per year.

\section{Multi-target Sequence Optimization}

From a fixed database, grouping the PO vectors referring to potential target debris, it is possible to suitably modify the optimizer, in order to compute the minimum $\Delta V$ associated with the rendezvous sequence, while fixing the time of flight at a constant value of 1,000 . This can be done by adding up a dimension to the POPULATION vector, with the 7th dimension containing the information about the optimal target to be reached:

$$
\begin{gathered}
\text { POPULATION }= \\
{\left[t_{1}-t_{0}, t_{3}-t_{2}, \Delta V_{1}, \Delta V_{2}, \varphi_{1}, \varphi_{2}, \text { target }\right]}
\end{gathered}
$$

With a potential set of $N$ debris to be captured in a sequence, it is possible to apply the optimizer, with $N$ - 1 simulations in succession. The orbital parameters of the chaser in step $N$ correspond to those of the target reached in the previous step $N-1$ (except for the value of true anomaly and the maneuver start time, which need to be updated for all objects, of a value corresponding to the time of flight of each new step). The calculation of the optimal sequence in this case will be limited to a single depth analysis, that is the choice of the target for the step $N$ is made exclusively 


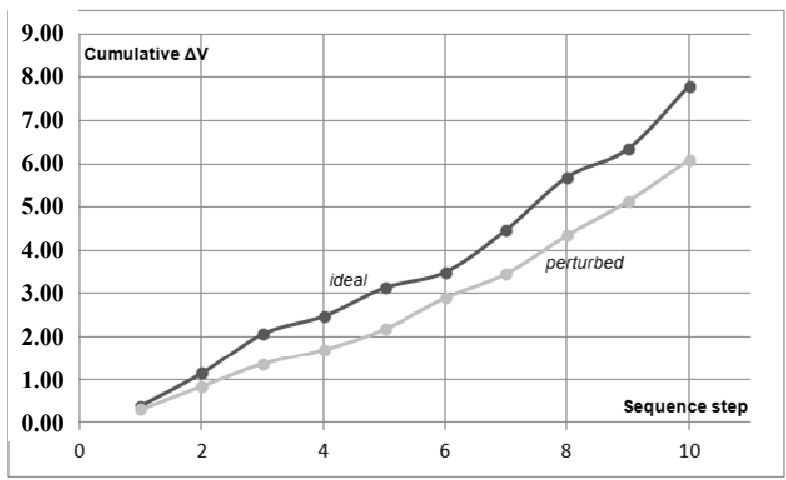

Fig. 6 Multi-target rendezvous sequence cumulative energy for the ideal and $J_{2}$ perturbed case.

on the basis of the positions of the entire population in that step, without taking into account possible advantages occurring in future stages. Consequently at each step the optimizer provides the selection of the target to which is associated with the least rendezvous $\Delta V$ at that moment. In reality, however, it may be advisable to reach a target that is associated with a slightly higher $\Delta V$, but which then leads to being in a better position to make the next rendezvous. If the depth of analysis is equal to the number of objects in the sequence, the global optimum is reached. A problem of this kind is very similar to the so-called Travel Salesman Problem [9]. It is clear that for numerous populations of debris, the effort required to compute the global optimal sequence is not sustainable. In this study, it was therefore adopted a single-depth strategy. Finally, ten randomly selected objects have been used to perform a comparison between ideal and perturbed cases.

From the Fig. 6, it can be noted that the $J_{2}$ effect contributes to a significant $\Delta V$ saving of more than $20 \%$, associated with the rendezvous sequence of the 10 objects.

\section{Conclusions}

Multi-target rendezvous applied to debris capture are gaining a growing interest, in perspective of the implementation of ADR techniques, which are necessary for a proper control of LEO debris hazard. Optimal number of objects to be removed each year is still under evaluation and future research may better address the extent and range of typical ADR reference missions. In the present study, it was taken into account a reference value of 10 objects to be removed per year. The main objective was then to optimize a rendezvous sequence in LEO, by exploiting the $J_{2}$ perturbation effect on both chaser and target orbital parameters ( $\Omega$ and $\omega$ ), directly affected by the disturbance. Every maneuver composing the final sequence has been modeled as a 4-impulses time fixed rendezvous, with the objective to minimize $\Delta V$ associated with each single step.

$J_{2}$ disturbance may be advantageous only for some combination of $\Omega$ and $\omega$, so the target shall be accurately selected at the beginning of each new step in the sequence. Nevertheless a debris population with random distribution of $\Omega$ and $\omega$ ensures the possibility to always be able to find a proper combination of parameters. The optimization strategy involved the use of a hybrid evolutionary algorithm, that was set up in order to search for an optimal solution, expressed by a $7 \mathrm{D}$ vector, whose first six components uniquely define the single rendezvous maneuver, whereas the last one identifies the target to be reached.

Simulations run in the frame of the study clearly showed a sensible $\Delta V$ saving on the final sequence, when $J_{2}$ effect is activated in the optimizer. Yet the optimal sequence finally found is not a global optimum, since the optimizer acts only in a single depth search, whereas the global optimum involves a $\mathrm{N}$-depth optimization strategy with $\mathrm{N}$ referring to the number of objects to be captured in the sequence. Further efforts in this field may be therefore addressed towards the increase of analysis depth, taking also into account target positions at times greater than the single rendezvous fixed time of flight.

In the frame of industrial R\&D, CADET project, currently carried on at Airbus Defence \& Space, is primarily aimed to develop an optimal capture mechanism for a single rendezvous maneuver, with the possibility to extend the range of potential targets 
up to six per mission. This reflect the need to progress in the field of target autonomous recognition and non-cooperative docking, before being able to plan multi-target missions, which will be essential to guarantee a safe and stable orbital environment.

\section{References}

[1] UN. 1999. Scientific and Technical Subcommittee of the United Nations Committee on the Peaceful uses of Outer Space. Technical report on space debris, New York.

[2] NASA (National Aeronautics and Space Administration), 2008. Handbook for Limiting Orbital Debris. Washington D.C.: NASA.

[3] Kessler, D. J., Johnson, N. L., Liou, J. C., and Matney, M. 2010. "The Kessler Syndrome: Implications to Future Space Operations." 33rd Annual AAS Guidance and Control Conference, Breckenridge, Colorado.
[4] Liou, J. C., Johnson, N. L., and Hill, N. M. 2010. "Controlling the Growth of Future LEO Debris Population with Active Debris Removal." Acta Astronautica 66 (5-6): 648-53.

[5] Liou, J. C., Hall, D. T., Krisko, P. H., and Opiela, J. N. 2004. "LEGEND, a 3-Dimensional LEO to GEO Debris Evolutionary Model.” Adv. Space Res.

[6] Sentinella, M. R. 2008. "Development of New Procedures and Hybrid Algorithms for Space Trajectory Optimization." Ph.D. thesis, Politecnico di Torino.

[7] Sentinella, M. R., Casalino, L. 2009. "Cooperative Evolutionary Algorithm for Space Trajectory Optimization." Celestial Mechanics and Dynamical Astronomy Journal, 105: 211-27.

[8] NORAD, 2012, Two-Line Element Sets, accessed October 2012, http://clestrak.com/.

[9] Barbee, B. W., Davies, G. W., Hur-Diaz, S. 2009. "Spacecraft Trajectory Design for Tours of Multiple Small Bodies." AAS (Advances in the Astronautical Sciences), 135: 2169-88. 